\title{
Respiratory syncytial virus contributes to more severe respiratory morbidity than influenza in children $<2$ years during seasonal influenza peaks
}

\author{
Rachid Amini ${ }^{1}\left[\right.$. Rodica Gilca ${ }^{1,2,3} \cdot$ François D. Boucher ${ }^{2,3} \cdot$ Hugues Charest ${ }^{1} \cdot$ Gaston De Serres $^{1,2,3}$
}

Received: 26 October 2018 / Accepted: 19 February 2019 / Published online: 23 February 2019

(c) Springer-Verlag GmbH Germany, part of Springer Nature 2019

\begin{abstract}
Purpose To compare the frequency and the severity of influenza and respiratory syncytial viruses (RSV) infections among children $<24$ months hospitalized with respiratory symptoms.

Methods Data from a prospective study conducted during the peak of five influenza seasons in the Province of Quebec, Canada were used.

Results We detected higher frequency of RSV compared to influenza viruses (55.3\% vs. 16.3\%). Radiologically confirmed pneumonia was significantly more frequent in children with RSV (39\%) than those with influenza (18\%) and the clinical course was more severe in RSV than influenza-infected children, especially among infants $<3$ months.

Conclusion Even during peak weeks of influenza season, we found a higher burden and severity of RSV compared with influenza virus disease in hospitalized children $<24$ months.
\end{abstract}

Keywords Influenza $\cdot$ Respiratory syncytial virus $(\mathrm{RSV}) \cdot$ Detection $\cdot$ Hospitalization $\cdot$ Children

\section{Introduction}

Respiratory syncytial virus (RSV) and influenza virus infections are considered as leading cause of hospitalization among infants and young children [1,2]. In a recent study, the average hospitalization rate attributable to RSV infection has been reported to be 5.2 per 1000 children under 2 years with seasonal variation from 2.5 to 9.9 per 1000 children [3]. Among the same age group, a systematic review has estimated influenza-associated hospitalization rates from 1 to 5 per 1000 children [1]. For both viruses, the highest hospital admission rates were among infants $<3$ months with an average rate of 17.9 and 3.3 per 1000 children, respectively, for RSV and influenza [3, 4]. Most studies assessing the influenza and RSV-associated hospitalization burden focus on the entire respiratory viruses season. Little information is

\section{Rachid Amini}

rachid.amini@inspq.qc.ca

1 Institut National de Santé Publique du Québec, 2400 Avenue d'Estimauville, Quebec, QC G1E 7G9, Canada

2 CHU de Quebec, Quebec, QC, Canada

3 Laval University, Quebec, QC, Canada available about the role of influenza viruses relative to RSV during periods of high influenza circulation among young children. The aim of this study is to compare the frequency and disease severity of influenza and RSV infections among children $<24$ months hospitalized with respiratory symptoms during the peaks of five influenza seasons.

\section{Methods}

In the province of Quebec, Canada, a prospective surveillance study with virologic assessment for influenza and other respiratory viruses was conducted during the peak weeks of influenza circulation among patients admitted for acute respiratory symptoms to four acute-care hospitals since 2012-2013. Peak weeks are defined as consecutive weeks with $\geq 15 \%$ of weekly specimens testing positive for influenza as monitored by the provincial sentinel laboratory network. All patients hospitalized for fever/feverishness or cough or sore throat have a nasal specimen collected with a flocked swab. Specimens are tested by the Luminex xTAG ${ }^{\circledR}$ RVP FAST multiplex, a polymerase chain reaction (PCR) assay which can detect 17 respiratory viruses (RV) [5]. Patients admitted for $\geq 24 \mathrm{~h}$ to the hospital floor are invited 
to participate in the study; those who give their informed consent are included. Demographic and clinical information of patients including chronic conditions associated with increased risk of influenza complications is collected using a standardized questionnaire. In our study, patient's chronic conditions information was retrieved by nurses from medical records and we did not ascertain diagnosis criterions of the asthma condition identified through the chart review among the children $<2$ years. As it is difficult to diagnose asthma in these young children and confirm it as definite bronchial asthma, we used the term "asthma-like condition". Additional details are presented elsewhere [5]. The peak weeks of the 2012-2013 and 2014-2015 to 2017-2018 influenza seasons were included in the analysis. The 2013-2014 season was excluded, because only influenza viruses were tested during this season. Proportions were compared with the Chi-square test or the Fisher's exact test where appropriate. Continuous variables were compared using Wilcoxon and Kruskal-Wallis nonparametric tests.

\section{Results}

During the peaks of five influenza seasons lasting from 8 to 10 weeks, 546 children were enrolled in the study. Nearly, a third were infants $<3$ months $(34 \%, n=188), 15 \%$ were aged 3-5 months $(n=83)$ and $6-11$ months $(n=84)$, and $35 \%$ were aged $12-23$ months $(n=191)$. Except for the 2017-2018, where the epidemic peak was characterized by the concomitant circulation of influenza $\mathrm{A}$ and $\mathrm{B}$, all the other peak weeks of the study period corresponded mainly to the influenza A peak (Fig. 1). There was annual variation in RSV positivity over the peaks of five influenza seasons, with RSV activity increasing (2012-2013 and 2014-2015), declining (2015-2016), or overlapping influenza peak weeks through 2016-2017 and 2017-2018 influenza season (Fig. 1).

\section{Proportion of detected viruses}

At least one respiratory virus was identified in $94.1 \%$ of children. RSV was detected in more than half of children (55.3\%), and the presence of both influenza and RSV was observed in $1.6 \%$ of cases. Despite being during the peak weeks of the influenza epidemic, influenza viruses were detected in one of six children (16.3\%). Influenza A (H3N2) was the predominating subtype (60\%) and influenza A (H1N1) pdm09 and influenza B were detected in $24 \%$ and $15 \%$ of specimens, respectively. Other respiratory viruses (ORV) were detected in $20.9 \%$ of children: entero/ rhinoviruses $(7.1 \%)$, coronaviruses $(5.5 \%)$, human metapneumovirus (3.3\%) and parainfluenza viruses $(2.7 \%)$, and other viruses (adenovirus and bocavirus) (2.2\%). Among the young infants $<3$ months, the clinical course was less severe in human metapneumovirus $(n=3)$ than in RSV-infected children based on the presence of radiologically confirmed pneumonia ( $0 \%$ vs. 17\%), length of stay (median of 3 days vs. 4 days) and intensive care unit admission ( $0 \%$ vs. $8.6 \%$ ), although differences are not statistically significant due to the small sample size. RSV was more frequently detected among infants aged 3-5 months $(73.5 \%)$ than in those $<3$ months (53.7\%), 6-11 months (53.6\%), and 12-23 months (49.7\%) $(p=0.003)$. Conversely, influenza was more frequent among older children aged $12-23$ months $(22.5 \%)$ than in those $<3$ months (14.4\%), 3-5 months (8.4\%), and 6-11 months $(14.3 \%)(p=0.02)$.

\section{Patient's characteristics by influenza and RSV detection}

There was no significant difference by age group in high-risk conditions for influenza complications, consultation delay, or influenza vaccine use between influenza and RSV-infected children (Table 1). The distribution of the main reasons for admission was similar between influenza and RSV infections among children aged 3-5 months and 12-23 months. Among infants $<3$ months, influenza-infected cases were more often admitted for fever than RSV patients (74\% vs. $26 \%$ ), while patients with confirmed RSV were more frequently hospitalized for dyspnea or bronchiolitis compared to influenza patients.

The 2014 WHO severe acute respiratory illness (SARI) case definition for influenza surveillance was met by more than $70 \%$ of children with influenza or RSV infections, with a higher proportion (83-89\%) among children $\geq 6$ months than youngest children and no significant difference between influenza and RSV-infected patients (Table 1). Fever (reported or measured) and cough were the two symptoms included in this case definition. Infants $<3$ months with influenza had significantly higher rate of fever than those with RSV $(100 \%$ vs. $63 \%, p<0.0001)$ and significantly less cough $(54 \%$ vs. $99 \%, p<0.0001)$. We did not found in our analysis any specific sign or symptom discriminating between influenza and RSV infection in children $<24$ months. Among these children, those with RSV had a significantly higher proportion of radiologically confirmed pneumonia than influenza patients ( $39 \%$ vs. $18 \%, p<0.01)$ (Table 1).

\section{Hospital course by influenza and RSV detection}

The median length of stay (LOS) in <24-month-old children was similar ( 3 days) for both influenza and RSVinfected children. Among infants $<6$ months, median LOS was longer for RSV (4 days) than influenza ( 3 days, $p<0.0001)$. While no child with influenza was admitted to 

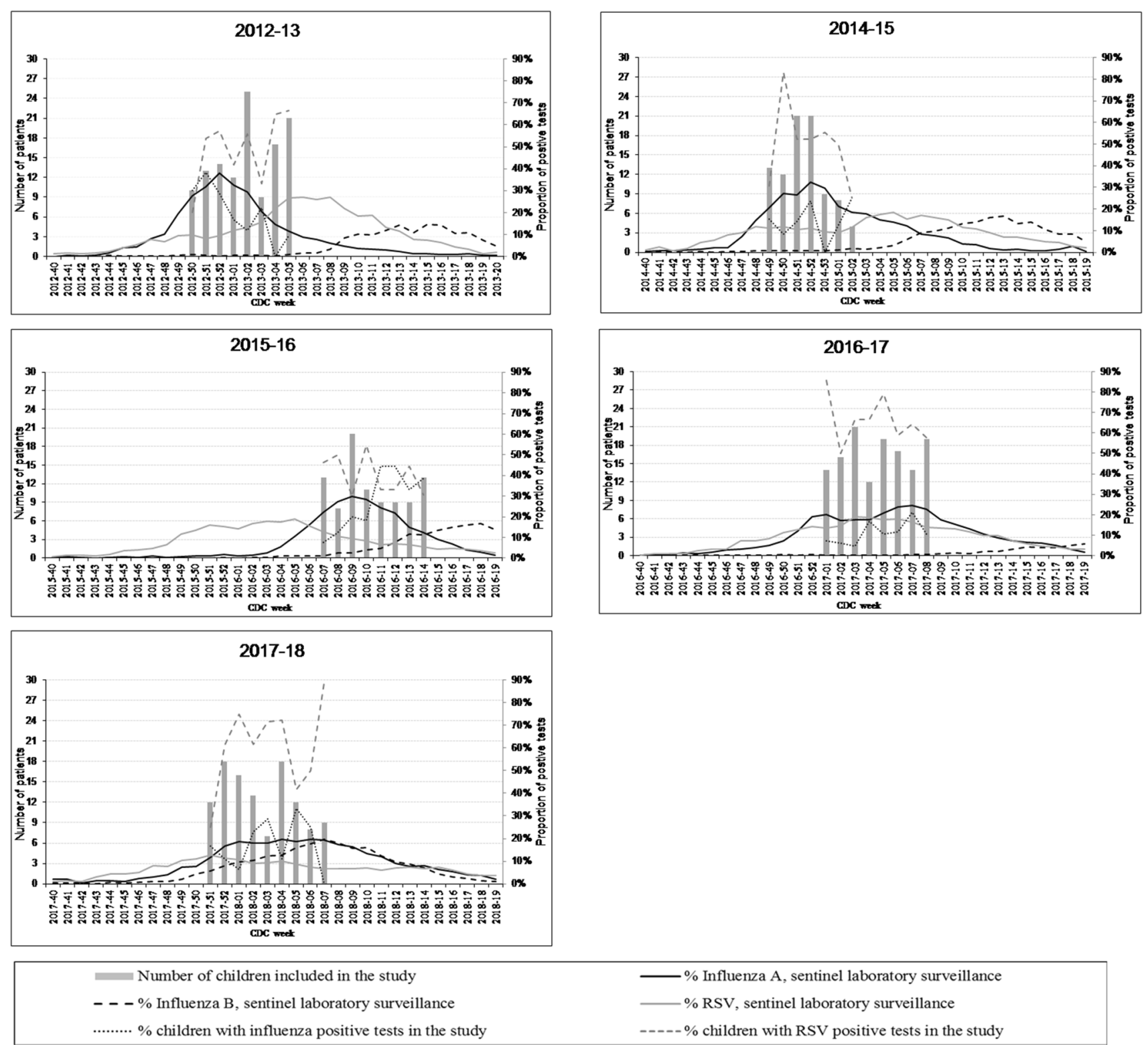

Fig. 1 Number of children enrolled in the study and proportion of influenza and RSV positive tests in the study and Quebec sentinel laboratory surveillance by $\mathrm{CDC}$ weeks and influenza season. $R S V$ respiratory syncytial virus

the intensive care unit (ICU), 3.6\% ( $n=11)$ of RSV-infected children required it and most of them $(82 \%, 9 / 11)$ were aged $<3$ months (Table 1). No death was reported among enrolled children.

\section{Discussion}

During peak weeks of influenza season with mostly no overlapping RSV peak weeks, we found a significantly higher burden of RSV compared with influenza virus disease in hospitalized children $<24$ months. Our results underline the importance of testing young children with respiratory symptoms for a panel of respiratory viruses and the limited value of etiologic diagnoses based on clinical definition and epidemiologic data. In addition, our findings of a substantially greater RSV frequency among hospitalized young children even during influenza peak weeks underscore the need of prevention and control approaches that address RSV infections.

To the best of our knowledge, this is the first study that describe the role of RSV relative to influenza viruses during the peak weeks of influenza epidemics among hospitalized young children. Other prospective surveillance studies from the United States and Europe documented the burden of influenza and RSV among enrolled children during whole 


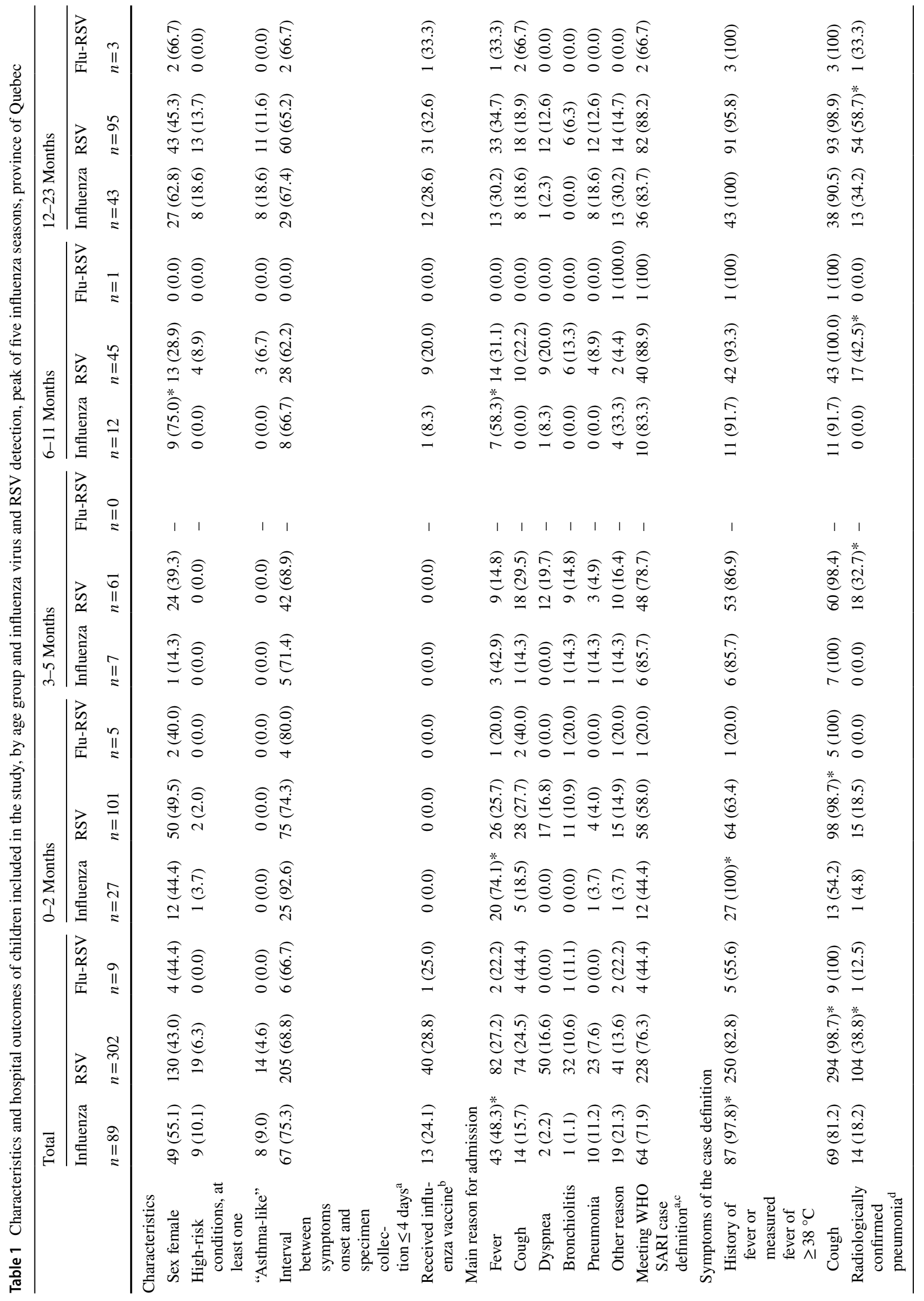


influenza seasons or calendar years [6-8]. Conducted mainly among children $<5$ years in early 2000 s, these studies reported similar patterns of high RSV detection (20-38\%) relative to influenza (3-15\%). A recent study from South Africa reported that during a 5-year period (2011-2016), RSV was more often detected (24\%) than influenza viruses $(6 \%)$ in children $<5$ years [9]. We found the highest rate of RSV among infants aged 3-5 months with RSV detection in nearly three over four patients $(73.5 \%)$ followed by infants $<3$ months (53.7\%). Similar to our results, other reports found that infants aged 3-5 months had the highest proportion of RSV detection [3, 10], while the highest RSV frequency in the South African study was among infants $<3$ months [9].

The clinical presentation of RSV infection in our study revealed the predominance of cough over the fever in particular among infants $<3$ months. Conversely, young infants infected with influenza had a significantly higher rate of fever than children with RSV infection. These results are consistent with previous reports on the RSV and influenza clinical features in young children $[8,9]$. We also found that a radiologically confirmed pneumonia was more frequent in RSV infection than in influenza infection, which is in line with other publications $[7,8]$. There was no difference in the median hospital duration ( 3 days) between RSV and influenza-infected children $<2$ years, a finding similar to the South African study that reported a non-significantly different median LOS (4 days) for RSV and influenza-infected children $<5$ years [9]. However, there were differences by age group in our study: infants $<6$ months hospitalized with RSV infection had significantly longer hospital stay compared to those with influenza infection (median LOS of 4 days vs. 3 days). A longer average duration of hospitalization among RSV-infected infants has also been previously reported [7, 11].

In this study, RSV infection was associated with higher but not significantly different ICU admission rates than influenza infection $(3.6 \%$ vs. $0 \%, p=0.07)$ among children $<2$ years. A comparable rate of $3.1-4.3 \%$ of ICU admissions associated with RSV has been reported in other prospective studies $[6,9]$. There were no deaths among children included in our analysis, which is consistent with recent studies reporting the relatively low case-fatality rate $(<1 \%)$ associated with pediatric influenza or RSV infections [12, 13].

There are several limitations to this study. First, RSV seasonal epidemics overlapped differently with epidemic curves of influenza during the study period. Both adults and children contribute to the Quebec provincial sentinel data for respiratory viral surveillance. It is likely that children disproportionately drive RSV findings in the community and conversely that adults predominate influenza circulation data. Even if RSV and influenza circulation in 
both adults and children may not coincide exactly with that of children, the overall RSV circulation relative to that of influenza is likely to be reflected in the sentinel surveillance curves we have presented. Second, we defined influenza peak weeks as those during which the percentage of positive specimens tested was $\geq 15 \%$ as monitored by the provincial sentinel laboratory network. This definition was based on provincial data indicating that weeks with $\geq 15 \%$ of influenza-positive specimens represented more than $74 \%$ of total influenza-positive tests during entire influenza season, and this peak week definition was used in line with previously published works $[14,15]$. During the peak influenza weeks of the 5 year study period, RSV and influenza had varying degree of seasonal overlap (Fig. 1). Relatively complete RSV and influenza peak weeks' overlap was evident during the two last seasons (2016-2017 and 2017-2018), with more than half (56\%) of the total of RSV positive tests during the peak influenza included in our study. However, after excluding these two seasons, we found similar results of higher frequency of RSV compared to influenza infections (data not presented). Third, there was no information about RSV risk factors for hospitalization such as history of prematurity, specific comorbidity (cystic fibrosis, neuromuscular diseases, etc.) as our original data focused on depicting the epidemiology of influenza infections. The low frequency of influenza detection is unlikely due to vaccination given the low rate of influenza vaccination among pregnant women $(\sim 16 \%)$ and children <2 years (17-19\%) in Quebec [16, 17], and little evidence is available on effectiveness of current influenza vaccines for children $<2$ years [18]. Finally, influenza antiviral treatment data were not recorded systematically all seasons, preventing us from analyzing its impact on influenza duration of hospitalization.

In conclusion, even during the peak weeks of influenza, more than half of hospitalizations for respiratory infections in children $<2$ years of age was due to RSV, with a clinical course more severe than influenza notably among infants $<3$ months.

\section{Compliance with ethical standards}

Conflict of interest This work was a part of a study supported by the Ministère de la Santé et des Services sociaux du Québec. R. G. received research funding from Ministère de la Santé et des Services sociaux du Québec for this study and research grants from Sanofi Pasteur and Pfizer for unrelated studies. All other authors report no potential conflicts.

Ethical statement Institutional review board approval was obtained from all participating hospitals for the first two years; an exemption was obtained for the rest of years given the change of the design and objectives from a research study into a surveillance project.

\section{References}

1. Nair H, Brooks WA, Katz M, Roca A, Berkley JA, Madhi SA, et al. Global burden of respiratory infections due to seasonal influenza in young children: a systematic review and metaanalysis. Lancet. 2011;378:1917-30.

2. Shi T, McAllister DA, O'Brien KL, Simoes EAF, Madhi SA, Gessner BD, et al. Global, regional, and national disease burden estimates of acute lower respiratory infections due to respiratory syncytial virus in young children in 2015: a systematic review and modelling study. Lancet. 2017;390:946-58.

3. Hall CB, Weinberg GA, Blumkin AK, Edwards KM, Staat MA, Schultz AF, et al. Respiratory syncytial virus-associated hospitalizations among children less than 24 months of age. Pediatrics. 2013;132:e341-8.

4. Chaves SS, Perez A, Farley MM, Miller L, Schaffner W, Lindegren ML, et al. The burden of influenza hospitalizations in infants from 2003 to 2012, United States. Pediatr Infect Dis J. 2014;33:912-9.

5. Gilca R, Amini R, Douville-Fradet M, Charest H, Dubuque J, Boulianne $\mathrm{N}$, et al. Other respiratory viruses are important contributors to adult respiratory hospitalizations and mortality even during peak weeks of the influenza season. Open Forum Infect Dis. 2014; 1:ofu086.

6. Iwane MK, Edwards KM, Szilagyi PG, Walker FJ, Griffin MR, Weinberg GA, et al. Population-based surveillance for hospitalizations associated with respiratory syncytial virus, influenza virus, and parainfluenza viruses among young children. Pediatrics. 2004;113:1758-64.

7. Forster J, Ihorst G, Rieger CH, Stephan V, Frank HD, Gurth H, et al. Prospective population-based study of viral lower respiratory tract infections in children under 3 years of age (the PRI. DE study). Eur J Pediatr. 2004;163:709-16.

8. Wolf DG, Greenberg D, Kalkstein D, Shemer-Avni Y, GivonLavi N, Saleh N, et al. Comparison of human metapneumovirus, respiratory syncytial virus and influenza A virus lower respiratory tract infections in hospitalized young children. Pediatr Infect Dis J. 2006;25:320-4.

9. McMorrow ML, Tempia S, Walaza S, Treurnicht FK, Moyes $\mathrm{J}$, Cohen AL, et al. The role of HIV in influenza- and respiratory syncytial virus-associated hospitalizations in South African children, 2011-2016. Clin Infect Dis. 2018. https://doi. org/10.1093/cid/ciy532.

10. Fryzek JP, Martone WJ, Groothuis JR. Trends in chronologic age and infant respiratory syncytial virus hospitalization: an 8-year cohort study. Adv Ther. 2011;28:195-201.

11. Resch B, Eibisberger M, Morris N, Müller W. Respiratory syncytial virus- and influenza virus-associated hospitalizations in infants less than 12 months of age. Pediatr Infect Dis J. 2011;30:797-9.

12. Bont L, Checchia PA, Fauroux B, Figueras-Aloy J, Manzoni P, Paes B, et al. Defining the epidemiology and burden of severe respiratory syncytial virus infection among infants and children in Western countries. Infect Dis Ther. 2016;5:271-98.

13. Tran D, Vaudry W, Moore D, Bettinger JA, Halperin SA, Scheifele DW, et al. Hospitalization for Influenza A Versus B. Pediatrics. 2016. https://doi.org/10.1542/peds.2015-4643.

14. Kuster SP, Kuster SP, Drews S, Green K, Blair J, Davis I, Downey J, et al. Epidemiology of influenza-associated hospitalization in adults, Toronto, 2007/8. Eur J Clin Microbiol Infect Dis. 2010;29:835-43.

15. Kuster SP, Katz KC, Blair J, Downey J, Drews SJ, Finkelstein S, et al. When should a diagnosis of influenza be considered in adults requiring intensive care unit admission? Results of populationbased active surveillance in Toronto. Crit Care. 2011;15:R182. 
16. Legge A, Dodds L, Macdonald NE, Scott J, McNeil S. Rates and determinants of seasonal influenza vaccination in pregnancy and association with neonatal outcomes. CMAJ. 2014;186:E157-64.

17. Kiely M, Boulianne N, Ouakki M, Diane Audet M-C, Gariepy, et al. Enquête sur la couverture vaccinale des enfants de 1 an et 2 ans au Québec en 2016. Institut national de santé publique du Québec; 2017. https://www.inspq.qc.ca/publications/2341. Accessed 10 Oct 2018

18. Jefferson T. Rivetti A, Di Pietrantonj C, Demicheli V. Vaccines for preventing influenza in healthy children. Cochrane Database Syst Rev. 2018. https://doi.org/10.1002/14651858.CD004876.pub4. 